\section{Microwave Myths and Tissue Processing}

\author{
Phillip McArdle \\ Energy Beam Sciences, East Granby, CT \\ PMcardle@ebsciences.com
}

Microwave-assisted preparation of histological samples has been performed for decades; what began with a few pioneering researchers has now become a routine and accepted practice in many clinical and research laboratories. Reliable, reproducible microwave protocols have been developed for a variety of operations: LM and EM processing, decalcification, fixation, special stains, antigen retrieval and more. Laboratories employing microwave procedures often do so for several compelling reasons: in addition to the expected time savings (often on the scale of orders of magnitude), improved morphology, retained immunoreactivity, and the elimination of hazardous reagents are benefits typically realized as well.

Despite the increasing availability of laboratory microwaves, consumer-grade ("kitchen") microwaves continue to be used, almost invariably due to cost considerations. (EBS has maintained since 1992 that a kitchen microwave has no place in the lab.) At any time in the US there are hundreds of microwave models to choose from: a dizzying array of sizes, wattages, options, and configurations await the shopper. This selection may be wonderful (if daunting) for a cook, but a nightmare for laboratory standardization. Therefore, although some users have achieved varying levels of success in some "non-tissue-processing" applications like staining, a high number of failures served to regress the art via anecdotal microwave "horror stories."

For these and other reasons, as protocols were developed and placed into use over the years, some myths, misconceptions, and just plain "bad science" began to creep in. Today, many highly educated and respected professionals use microwaves in their lab, occasionally blissfully unaware of the mechanisms at work, or the fact that they are using outdated protocol or equipment when more effective alternatives are available.

\section{Common questions and misconceptions}

Microwaves do not heat from the inside out. The energy has to penetrate material from the outside in, so the material heats from the outside in; exposure decreases with depth. However, due to their small size, histological samples usually heat at the same rate throughout.

A microwave "load" is anything in the microwave that converts microwaves to heat. It's important that a sufficient load be used, or equipment and/or tissue damage can occur. Basically, the microwave energy has to go somewhere; if there's an insufficient load, small or slightly microwave absorbent material can absorb excessive energy.

Microwaves do not heat all material equally. Microwaves heat polar material like water and alcohols, and don't heat non-polar material like alkanes. Some fats, being emulsions or other associations with polar components, do heat. Materials like Pyrex can be slightly polar and can absorb microwave energy. And a metal can cause "arcing" (sparking).

"Microwave transparency" isn't synonymous with "optical transparency." It means the material allows the passage of microwaves without converting a significant portion to heat. Many materials that we wouldn't think of as "transparent," like opaque white Teflon are, in fact, "microwave transparent." How to determine microwave trans- parency: Fill a glass container with approximately $50 \mathrm{ml}$ of water. Place in the microwave next to the (empty) container in question. Set the microwave on maximum power and heat for one minute. If the new container is warm or hot, it is absorbing microwave energy; if it remains at room temperature, it is microwave transparent.

EBS strongly discourages microwaving formalin in any form, since there are serious health-and-safety issues involved. However, we recognize that safe and effective alternatives to reagents like zinc formalin have yet to be proven in some cases, so formalin can be a "necessary evil." If it is necessary to use formalin (like zinc formalin for decalcification), make sure that the temperature probe is kept clean of zinc build-up or arcing may result. And NEVER breathe warm formalin fumes!

Vacuum can be used in the embedding step when processing thick ( $>4 \mathrm{~mm})$, fatty, or thick and fatty samples. Too little vacuum will result in inadequate infiltration; too much can result in tissue damage like clefts. 15 " $\mathrm{Hg}$ is usually adequate, although up to 20 " $\mathrm{Hg}$ can usually be used.

Ideally, a laboratory microwave should have its own dedicated circuit, with nothing else plugged in. Anything with a heating element is one of the worst things to plug into the same circuit, since its high current draw can cause a severe drop in the microwave's magnetron output, producing inconsistent results.

Air agitation should almost always be used if available. Agitation promotes even heating of reagent, preventing uneven distribution of solutes, suspensions, etc. A good example is the pink meniscus ring that forms in microwave PAS staining procedures, when agitation is omitted. This layer rises to the top due to vaporization of $\mathrm{SO}_{2}$, and agitation helps prevent this. Rarely, a protocol may warn of the "damaging effects of bubbling," for example, but we have seen no evidence supportive of this claim and much evidence to the contrary.

It's important to prevent pressurization of microwave containers (with the exception of a pressure/vacuum vessel specifically designed for the relevant microwave application), so uncovered or vented containers are preferred. Paper toweling may be used as a light covering to prevent splatter and to absorb moisture. Waxed paper helps to retain heat and moisture.

Microwaves can superheat a reagent, causing explosive boiling when disturbed. This isn't exclusive to microwaves; it's essentially the same as "bumping" in a sand bath or Bunsen burner. However, boiling chips are not recommended.

Always...

- use a laboratory microwave for laboratory work

- connect the chamber venting system to an external fume removal system

- use manufacturer-approved containers

- use vented or uncovered containers when not using vacuum

- handle containers with potholders or thermal mitts

- use a fume hood to work with hazardous reagents such as formalin

- run controls

Periodically...

- use a microwave leakage detector to check for leakage

- inspect/clean chamber and hinges

- inspect/clean air intakes and filters (if so equipped); replace filter(s) as necessary

- inspect/clean temperature probe (if equipped) and probe mount

- inspect/clean door seal (if so equipped) and area where door and cabinet meet 


\section{Explore, discover, build \\ with unparalleled access to the nanoscale}

\section{FEI COMPANY}

TOOLS FOR

Today's advanced discovery and product development requires unimpeded 3D access to the nanoscale with the best resolution possible. FEI's Tools for Nanotech ${ }^{\mathrm{TM}}$ support the success of your most demanding projects by giving you the power you need to reach far into the nanoscale.

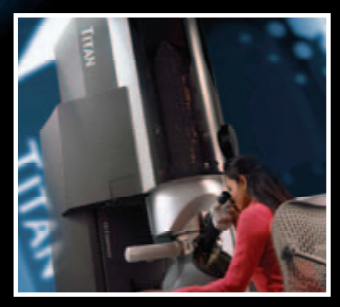

\section{Titan $^{\text {TM }}$ 80-300 (S)TEM}

The all-new Titan breaks the Ångström barrier with atomicscale resolution. This dedicated platform for aberration corrected (scanning) electron microscopy delivers the highest resolution available on a commercially available (S)TEM system.

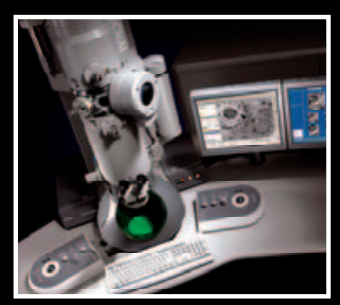

\section{Tecnai ${ }^{\mathrm{TM}} \mathbf{G}^{2}$ Series}

Any of the $G^{2}$ series offers a truly universal imaging and analysis solution that seamlessly embeds all peripherals and software in one powerful instrument. It offers total digital control of your advanced (S)TEM environment, and takes the proven Tecnai 'all-in-one' concept to a new level.

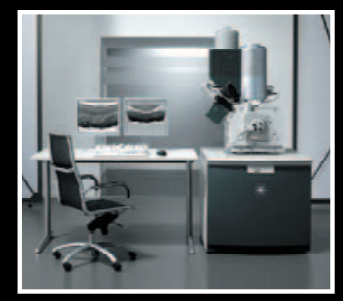

\section{Nova ${ }^{\text {TM }}$ NanoLab and Quanta ${ }^{\text {TM }}$ 3D}

FEI's range of market-leading DualBeam ${ }^{\text {TM }}$ (FIB/SEM) systems are optimized to give you the performance you demand for your specific applications range. FEI's Quanta 3D remains the gold standard for high-vac, low-vac and environmental 3D SEM applications.

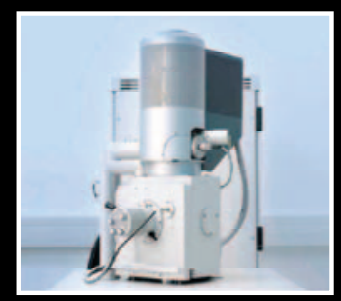

\section{Nova $^{\text {TM }}$ NanoSEM}

Experience the world's only true high-resolution, low-vacuum FEG-SEM for characterization of charging and/or contaminating nano-materials or nano-devices. 
- check samples (staining, processing, unmasking, etc.) against controls

Never...

- use a consumer-grade microwave for laboratory work

- cover containers tightly (with the exception of task-appropriate, specialized microwave vacuum or pressure vessels)

- operate the microwave without a load

- use metal accessories inside the unit

- heat food in a microwave oven used for laboratory procedures

- breathe warm reagent fumes, especially formalin

\section{New Microwave, Old protocol}

Knowing how to adapt old microwave protocols to new equipment can be a vital skill. Some old protocols were developed for an obscure "niche," while others have been used for years, on a daily basis, in countless clinical labs. Unfortunately, many were developed for use with kitchen microwaves lacking features now known to be essential in a laboratory context. Due to the variability of consumergrade microwaves, and their inadequate features, many creative work-arounds were developed that worked for one lab with the microwave they happened to own at that moment.

Unfortunately, work-arounds often resulted in additional steps, questionable settings, or odd instructions. But ultimately, any protocol can be reduced to simple elements. When faced with the task of updating an old protocol, it's best to try and determine the overall context; the specifications of the microwave used; power, time and temperature settings; and as many other useful details as possible. For example, when looking at the context of a given protocol, it may become apparent that a complicated, extensive string of specified temperatures may be merely a work-around to achieve a particular temperature at the end of the run, as a work-around for a microwave lacking a temperature probe.

At EBS we often are faced with something like this: "I have a very specific protocol that I want to use with my new lab microwave: 'Bring slides to boiling in a plastic Coplin jar. We use plastic containers that hold 24 slides, placed in vertically. We fill the container with $10 \mathrm{mM}$ Citrate $\mathrm{pH} 6.0$ buffer past the sections, almost to the top, leaving enough room so that the buffer will not boil over. We bring the slides to boiling. It takes 3 minutes on high power for us to reach this point. You want the temperature to just reach boiling. We then keep them at a sub-boiling temperature in the microwave for 10 minutes. To do this, we heat them at $30 \%$ power for $10 \mathrm{~min}$ utes (to keep them at $98-100^{\circ} \mathrm{C}$ ). We then remove the container from the microwave, leaving the top slightly ajar, we let them cool on the bench top."

It's easy to see that this "very specific" protocol is, in fact, anything but when it comes to the microwave:

Container: A Coplin jar is recommended, but is contradicted by specification of 24 slides.

Time and temperature: The desired temperature may be $99^{\circ} \mathrm{C}$, although $100^{\circ} \mathrm{C}$, "boiling," "sub-boiling" and $98^{\circ} \mathrm{C}$ are all specified. (Furthermore, we're actually concerned with the BP of buffer.) The first 3-minute setting appears to be merely "ramp up" time, the time required to reach the buffer's $B P$. It can safely be concluded that the microwave in question does not have a temperature probe.

Power: The microwave wattage is an unknown, so the $30 \%$ specification is of little help. Obviously if either the old microwave or detailed specifications are available, it's a simple matter to ascertain its wattage and calculate proper power settings for the new unit. In this case, we'll arbitrarily assume the original microwave was an 800watt model. $30 \%$ of 800 watts is 240 watts. If the new lab microwave is 1000 watts, $24 \%$ power gives the same 240 watts.

Overall, this confusing protocol can probably be reduced to: it's critical that the sections remain immersed in buffer that's just below boiling for 10 minutes. The first 3-minute step appears to be simple ramp-up time; since a good lab microwave can compensate for ramp-up time, this can be safely disregarded. Power appears to be important only insofar as it's being adjusted to maintain (probably) $99^{\circ} \mathrm{C}$, assuming that's almost at the BP of the buffer. Since a modern microwave processor measures temperature and adjusts its output to achieve a given temperature, power per se should be unimportant during the run. During ramp-up, however, excessive power might cause tissue damage, so it's a good idea to start off with low to moderate power settings. The Coplin jar recommendation can be ignored in favor of containers appropriate to hold 24 slides. Finally, it's safe to assume that agitation was unavailable; while we usually recommend agitation, to remain true to the original protocol, omit it during initial tests. Agitation can always be added later for comparison.

So for this protocol, first running controls and using non-critical samples, we would attempt 10 minutes @ 99 $\mathrm{C}$, start timer at setpoint temperature, $24 \%$ power, agitation off. To more faithfully replicate the original protocol, we would apply $80 \%$ power during ramp-up rather than $24 \%$ (this would require two preset steps), but this is probably unnecessary.

When replicating protocols developed for microwaves without a temperature probe, running a lab microwave into "power" mode would be equivalent. For example, special stain protocols often require less critical temperature control than microwave fixation, unmasking, and tissue processing. Or an unmasking protocol may have been designed for a pressure cooker with no provision for a temperature probe. Usually an ending temperature is specified, or can be found with some research. So, via trial and error, initially using water and then moving on to reagent and non-critical samples, the new microwave should be run in "power control" mode, and a power setting determined that provides the endpoint temperature at the proper time. Then, it may either be run in "power" mode, or if a temperature probe can be used, changed to "temperature" mode (retaining the power setting just tested) and the endpoint temperature programmed in. This should ensure that the desired temperature will not be exceeded.

\section{References}

Kok and Boon: Microwave Cookbook for Microscopists. Coulomb Press, Leyden 1992

Login, G.R., and Dvorak, A. M. (1994). The Microwave Tool Book. A Practical Guide for Microscopists. Boston: Beth Israel Hospital.

See Also

Clinical and Laboratory Standards Institute publication Microwave Device Use in the Histology Laboratory; Approved Guideline [ISBN 1-56238-563-1]

College of American Pathologists Publication ANP.29430

OSHA 29CFR 1910.303(b)(2)

Extra thanks to: Richard Dapson, Anatech Ltd., Battle Creek, MI, Denise Gerard, Thermo Electron Corporation, Pittsburgh, Pennsylvania, Vincent R. Klump, Jr., Histology Services, East Haven, CT, Joyce Moore, Jefferson Regional Medical Center Histo-Path Laboratories, Edison Narvaez, Greenwich Hospital, Greenwich, Connecticut, Donna Willis, Harris Methodist Hospital, Fort Worth, Texas.

In Memoriam: The late Steven Slap, former EBS colleague 


\section{Value and Excellence in SEMs}

VEGA Scanning Electron Microscopes

Fully automated PC-controlled SEMs designed for a multi-user environment.

High performance 4-lens optics provides high resolution, high depth of focus, extremely large field of view as well as optimum working conditions for any application.

High working vacuum and variable pressure operation up to $2000 \mathrm{~Pa}$.

Original patented Low Vacuum Secondary Detector for true secondary electron imaging under low vacuum.

Full range of precision-engineered specimen chambers and stages.

Sophisticated yet easy-to-use software for microscope control and image processing \&t evaluation.

Network operations with built-in remote control and diagnostics.

3D surface reconstruction using $3 \mathrm{D}$ beam technology.
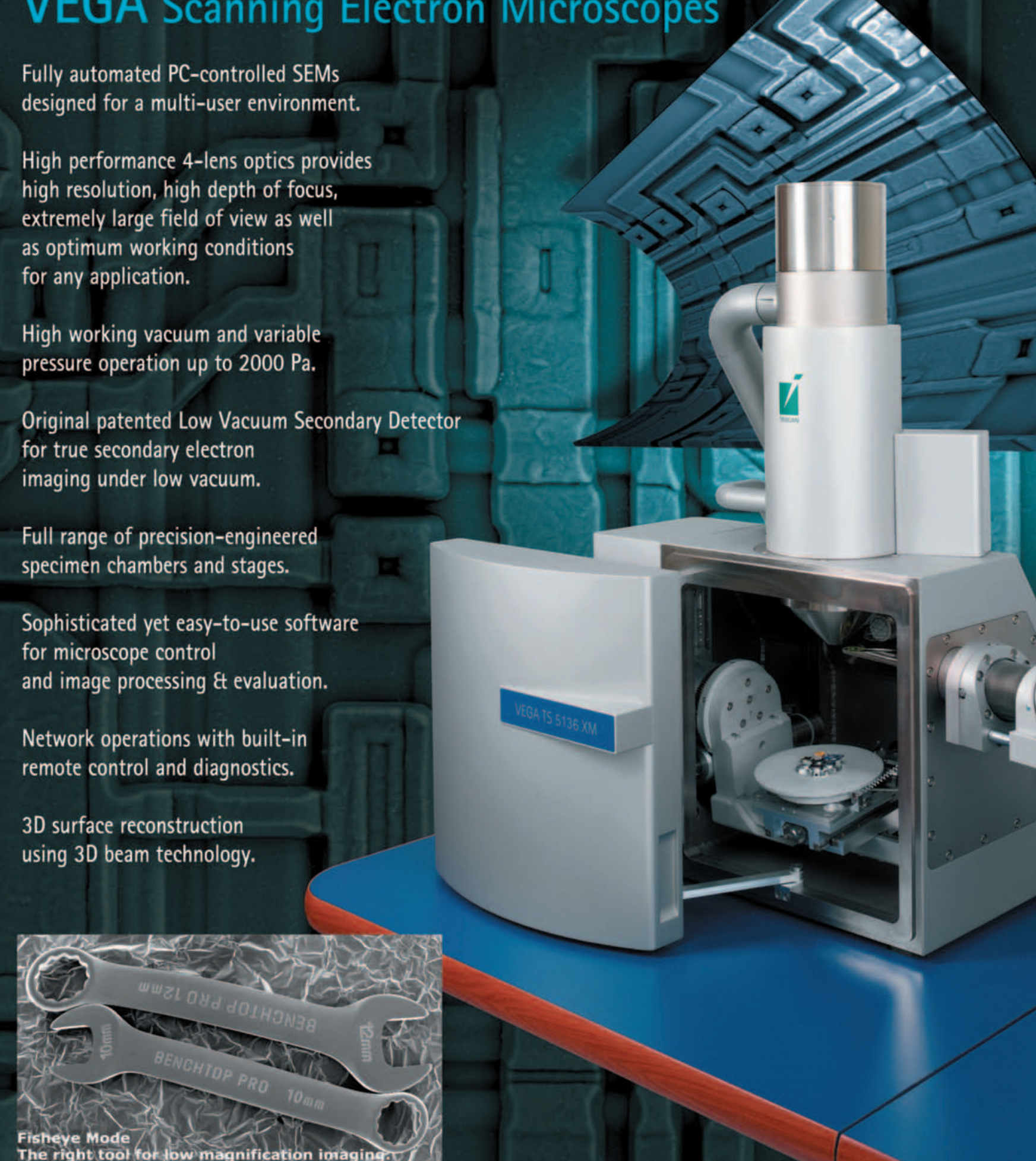